\title{
Plant growth regulators affect biosynthesis and accumulation profile of isoflavone phytoestrogens in high-productive in vitro cultures of Genista tinctoria
}

\author{
Maria Luczkiewicz • Adam Kokotkiewicz • \\ Daniel Glod
}

Received: 6 February 2014/Accepted: 18 April 2014/Published online: 30 April 2014

(c) The Author(s) 2014. This article is published with open access at Springerlink.com

\begin{abstract}
The influence of plant growth regulators on biomass growth and the accumulation of medicinally-relevant isoflavone phytoestrogens, derivatives of genistein and daidzein (8 compounds including aglycones, glucosides and glucoside esters) in callus cultures of Genista tinctoria (Fabaceae) was examined. The experiments included 10 auxins [2,4dichlorophenoxyacetic acid (2,4-D), p-chlorophenoxyacetic acid, indole-3-acetic acid, indole-3-butyric acid, indole-3-propionic acid, 1-naphthaleneacetic acid, $\beta$-naphthoxyacetic acid, picloram, 2,3,5-triiodobenzoic acid (TIBA), 2,4,5-trichlorophenoxyacetic acid $(2,4,5-\mathrm{T})$ ] and 7 cytokinins [6-benzylaminopurine, forchlorfenuron, 1,3-diphenylurea, 2-isopentenyladenine, kinetin (KIN), thidiazuron, zeatin] applied at 0.5 and $5.0 \mathrm{mg} \mathrm{l}^{-1}$, jointly with 5.0 or $0.5 \mathrm{mg} \mathrm{l}^{-1} \mathrm{KIN}$ or 2,4-D (for auxins and cytokinins, respectively-36 phytohormone combinations in total). Statistical analysis of the relationships between callus growth [expressed as growth index $(\mathrm{Gi})$ ] and the accumulation of isoflavones showed positive correlation in the cytokinin group $\left(\mathrm{r}_{\mathrm{xy}}\right.$ values from 0.13 to 0.61$)$ and negative correlation within auxins $\left(\mathrm{r}_{\mathrm{xy}}\right.$ values from -0.31 to -0.39 ). Among the cytokinins tested, the highest isoflavone content $(6,436.26 \mathrm{mg} / 100 \mathrm{~g}$ dry weight $)$ and the fastest biomass growth $(\mathrm{Gi}=892.46 \%)$ were obtained for $0.5 \mathrm{mg} \mathrm{l}^{-1} \mathrm{KIN}$ used jointly with $5.0 \mathrm{mg} \mathrm{l}^{-1} 2$,4-D. In the group of auxins, the combination of $0.5 \mathrm{mg}^{-1}$ TIBA and $5.0 \mathrm{mg}^{-1}$ KIN provided the fastest culture growth $(\mathrm{Gi}=983.07 \%)$ and the isoflavone concentration of $10,474.23 \mathrm{mg} / 100 \mathrm{~g}$ dry weight, which is so far the highest amount of these metabolites achieved in callus cultures of higher plants.
\end{abstract}

M. Luczkiewicz $(\bowtie) \cdot$ A. Kokotkiewicz · D. Glod Department of Pharmacognosy, Faculty of Pharmacy, Medical University of Gdansk, Al. Gen. J. Hallera 107, 80-416 Gdańsk, Poland

e-mail: mlucz@gumed.edu.pl
Keywords Auxins - Cytokinins - Daidzein - Genistein · Isoflavones · Phytoestrogens

\begin{tabular}{|c|c|}
\hline \multicolumn{2}{|c|}{ Abbreviations } \\
\hline BAP & 6-Benzylaminopurine \\
\hline 4-CPA & p-Chlorophenoxyacetic acid \\
\hline 4-CPPU & $\begin{array}{l}N \text {-(2-chloro-4-pyridyl)- } N^{`} \text {-phenylurea } \\
\text { (forchlorfenuron) }\end{array}$ \\
\hline 2,4-D & 2,4-Dichlorophenoxyacetic acid \\
\hline Di & Dynamics index \\
\hline 1,3-DPU & 1,3-Diphenylurea \\
\hline $\mathrm{Gi}$ & Growth index \\
\hline IAA & Indole-3-acetic acid \\
\hline IBA & Indole-3-butyric acid \\
\hline $2 \mathrm{iP}$ & $\begin{array}{l}\text { 6- }(\gamma, \gamma \text {-Dimethylallylamino)purine } \\
\text { (2-isopentenyladenine) }\end{array}$ \\
\hline IPA & Indole-3-propionic acid \\
\hline KIN & Kinetin \\
\hline NAA & 1-Naphthaleneacetic acid \\
\hline$\beta$-NOA & $\beta$-naphthoxyacetic acid \\
\hline PGR & Plant growth regulator \\
\hline PICL & Picloram \\
\hline $\mathrm{SH}$ & Schenk and Hildebrandt \\
\hline TDZ & $\begin{array}{l}N \text {-phenyl- } N \text { '-(1,2,3-thiadiazol-5-yl)urea } \\
\text { (thidiazuron) }\end{array}$ \\
\hline TIBA & 2,3,5-Triiodobenzoic acid \\
\hline $2,4,5-\mathrm{T}$ & 2,4,5-Trichlorophenoxyacetic acid \\
\hline ZEA & Zeatin \\
\hline
\end{tabular}

\section{Introduction}

Soy isoflavones, represented mainly by genistein, daidzein and their derivatives, belong to the most extensively 
investigated phenolics of plant origin. A wide interest in these compounds results from their multidirectional biological activity and associated health benefits, particularly related to cancer chemoprevention. Isoflavones act as selective estrogen receptor modulators, exhibiting agonist or antagonist activity depending on local estrogen concentrations (Fritz et al. 2013). They are also reported to affect multiple cellular signalling pathways, protect cells from oxidative damage and inhibit angiogenesis ( $\mathrm{Li}$ et al. 2011; Mahmoud et al. 2014). Numerous clinical trials indicate that regular consumption of isoflavone-rich foods may reduce the risk of breast and prostate cancers (Fritz et al. 2013; Mourouti and Panagiotakos 2013; van Die et al. 2014). Besides chemopreventive effects, major soybean isoflavones, genistein and daidzein, are considered promising agents for the treatment of diabetes, obesity and neurodegenerative diseases (Zhao et al. 2009; Behloul and Wu 2013).

The interest in isoflavones as potential therapeutic agents boosted research aimed at establishing biotechnological methods for their production, based chiefly on in vitro cultures of leguminous plants. Numerous cell lines were obtained, with notable examples including Glycine max (Federici et al. 2003), Genista sp. (Łuczkiewicz and Głód 2003; Tůmová and Tůma 2011), Pueraria sp. (Matkowski 2004; Goyal and Ramawat 2008; Udomsuk et al. 2009, 2012), Psoralea corylifolia (Shinde et al. 2010), Maackia amurensis (Fedoreyev et al. 2000, 2008; Luo et al. 2003, 2004, 2007), Trifolium pratense (Engelmann et al. 2009; Kašparová et al. 2012), Medicago truncatula (Lei et al. 2010) and Sophora japonica (Pu et al. 2013). Moreover, attempts are being made to improve isoflavone productivity of the obtained systems, either by means of genetic and molecular engineering (Du et al. 2010) or classical experimental techniques including clone selection and medium optimization (Luczkiewicz 2008). Among the latter, the modification of plant growth regulators (PGRs) combination is a simple yet effective tool for the regulation of biomass growth and different branches of secondary metabolism in plant cell cultures, including phenylpropanoid pathways (Luczkiewicz 2008; Matkowski 2008; Smetanska 2008). However, limited amount of data is so far available on the influence of PGRs on isoflavone production in plant in vitro cultures, with research focusing mainly on the effects of the most commonly used phytohormones on the accumulation of major isoflavone constituents (Vaishnav et al. 2006; Tůmová et al. 2007; Shinde et al. 2010).

The aim of the presented work was to examine the influence of different combinations of auxins and cytokinins on biomass growth and isoflavone content in dyer's broom (Genista tinctoria, Fabaceae) callus, which was previously shown to be a particularly rich source of genistein and daidzein derivatives (Łuczkiewicz and Głód
2003). As far as the authors are concerned, this is the first such comprehensive report on the phytohormone regulation of isoflavonoid metabolism in a single growth model, involving 17 PGRs in 36 combinations, tested for their effect on callus growth and accumulation of 8 constituents including isoflavone aglycones, glucosides and glucoside esters. The implications of the results for further isoflavone-related biotechnological studies are discussed.

\section{Materials and methods}

\section{Plant material}

The previously established (Łuczkiewicz and Głód 2003) callus cultures of $G$. tinctoria grown on solidified $(0.7 \%$ w/v agar) SH medium (Schenk and Hildebrandt 1972) containing $5.0 \mathrm{mg} \mathrm{l}^{-1}$ 2,4-Dichlorophenoxyacetic acid (2,4-D), $5.0 \mathrm{mg} \mathrm{l}^{-1}$ kinetin (KIN) and $3.0 \% \mathrm{w} / \mathrm{v}$ sucrose were used as a source of plant material.

Influence of PGRs on callus growth and accumulation of isoflavones

The effect of auxins on biomass growth and the accumulation of isoflavones in $G$. tinctoria callus cultures was investigated by enriching the KIN-supplemented $\mathrm{SH}$ media sequentially with 2,4-D, 1-Naphthaleneacetic acid (NAA), Indole-3-butyric acid (IBA), Indole-3-propionic acid (IPA), Indole-3-acetic acid (IAA), p-Chlorophenoxyacetic acid (4CPA), Picloram (PICL), $\beta$-naphthoxyacetic acid ( $\beta$-NOA), $2,4,5$-Trichlorophenoxyacetic acid $(2,4,5-\mathrm{T})$ and 2,3,5-Triiodobenzoic acid (TIBA). The media containing 5.0 and $0.5 \mathrm{mg} \mathrm{l}^{-1} \mathrm{KIN}$ were supplemented with 0.5 and $5.0 \mathrm{mg}^{-1}$ auxins, respectively. G. tinctoria calli grown on $\mathrm{SH}$ media supplemented with 0.5 or $5.0 \mathrm{mg} \mathrm{l}^{-1} \mathrm{KIN}$ only were considered as control samples ("C"-s. Fig. 1, Table 1).

In order to determine the effect of cytokinins on biomass growth and isoflavone accumulation, the experiment was conducted analogous to the one described above. The $\mathrm{SH}$ media containing 2,4-D were supplemented interchangeably with KIN, Zeatin (ZEA), 6-Benzylaminopurine (BAP), 6-( $\gamma, \gamma$-Dimethylallylamino)purine (2-isopentenyladenine) (2iP), $N$-(2-chloro-4-pyridyl)- $N$ - -phenylurea (forchlorfenuron) (4-CPPU), 1,3-Diphenylurea (1,3-DPU) and $N$-phenyl$N^{\prime}$-(1,2,3-thiadiazol-5-yl)urea (thidiazuron) (TDZ). The media containing 5.0 and $0.5 \mathrm{mg} \mathrm{l}^{-1}$ 2,4-D were supplemented with 0.5 and $5.0 \mathrm{mg}^{-1}$ cytokinins, respectively. $G$. tinctoria calli grown on SH media supplemented solely with 0.5 or $5.0 \mathrm{mg} \mathrm{l}^{-1} 2,4-\mathrm{D}$ were considered as control samples (Fig. 2, Table 1).

All experiments were conducted using solid $(0.7 \% \mathrm{w} / \mathrm{v}$ agar) $\mathrm{SH}$ media containing $3.0 \% \mathrm{w} / \mathrm{v}$ sucrose $(\mathrm{pH}$ adjusted 

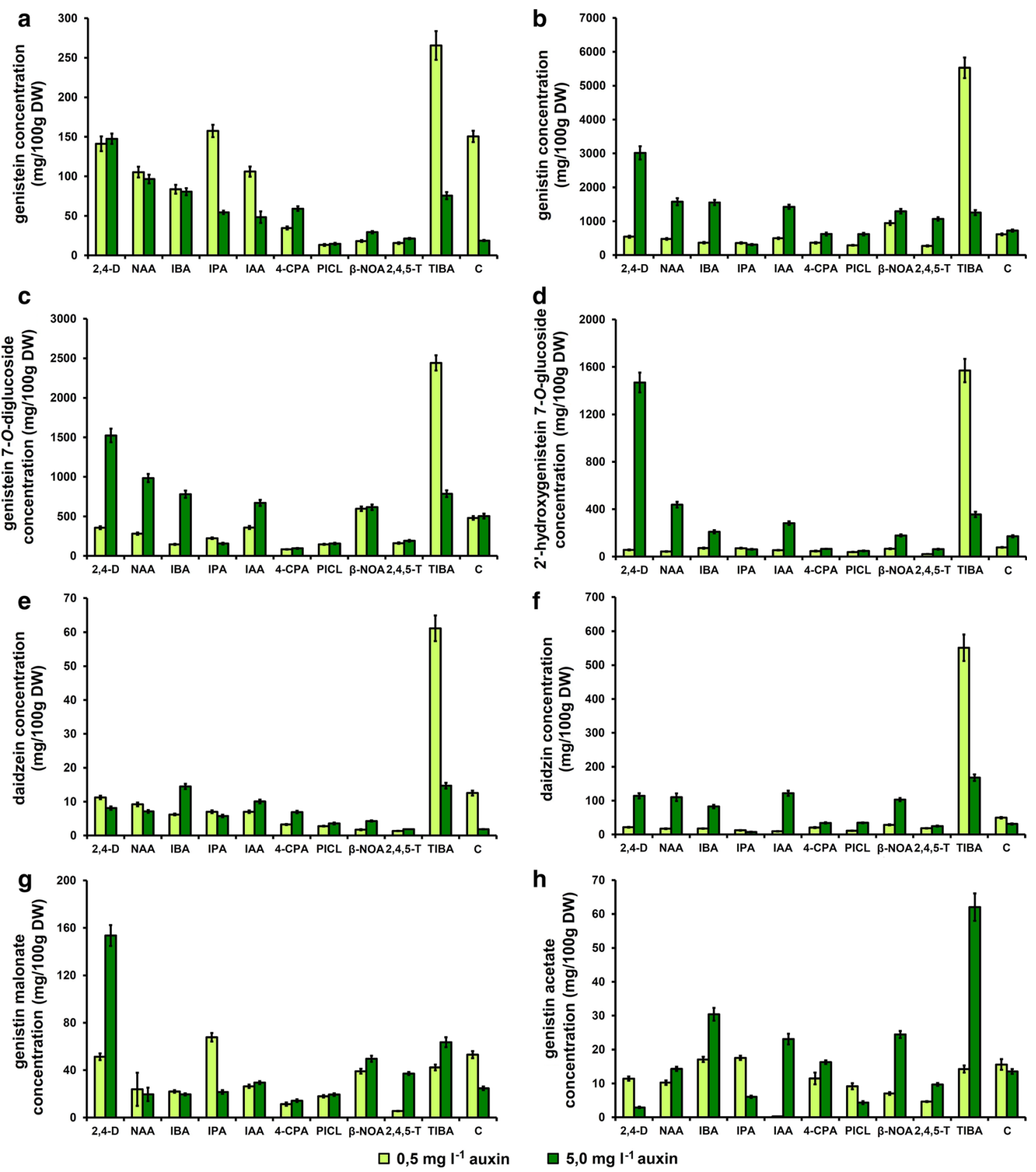

Fig. 1 The effect of various auxins and their concentrations on the accumulation of genistein $(\mathbf{a})$, genistin $(\mathbf{b})$, genistein 7- $O$-diglucoside (c), 2'-hydroxygenistein 7-O-glucoside (d), daidzein (e), daidzin (f),

genistin malonate (g) and genistin acetate (h) in Genista tinctoria callus. Values represent the mean \pm SD of 15 replicates

to 5.8 prior to autoclaving at $121{ }^{\circ} \mathrm{C}$ and 1 bar for $20 \mathrm{~min}$ ). The thermally unstable PGRs (IAA, TIBA, ZEA, 2iP, 4-CPPU, 1,3- DFM, TDZ) were added to the previously autoclaved media via sterile filtration through $0.2 \mu \mathrm{m}$ syringe filters (Schleicher \& Schuell/Whatman, SigmaAldrich, St. Louis, US-MO). The rest of the PGRs used were co-autoclaved with basic media ingredients (all reagents from Sigma-Aldrich; water produced using REL5 double water still, Mera-Polna, Przemysl, Poland).

For each medium modification, $300 \mathrm{mg}$ of fresh G. tinctoria callus was aseptically transferred into baby food jars (SigmaAldrich) containing $25 \mathrm{ml}$ of the experimental medium and closed with polypropylene caps ( 3 series of 5 jars, 15 samples in total). Callus cultures were maintained at $25 \pm 2{ }^{\circ} \mathrm{C}$ under continuous light $\left(88 \pm 8 \mu \mathrm{mol} \mathrm{m} \mathrm{m}^{-2} \mathrm{~s}^{-1}\right.$, Philips TLD $35 \mathrm{~W}$ fluorescent lamps). After a 4 -week period, the biomasses were collected and their growth indices (Gi) calculated using the formula proposed by Swedlund and Locy (1993)—Fig. 3. 
Table 1 The effect of SH media containing different concentrations of selected auxins and cytokinins on the biomass growth and isoflavone accumulation in G. tinctoria callus a $\mathbf{C}$ control group, $\uparrow \uparrow \uparrow$ more than threefold increase (Di $>3$ ), $\uparrow \uparrow$ two-threefold increase $(2 \leq \mathrm{Di} \leq 3)$, $\uparrow$ less than twofold increase $(1.05<\mathrm{Di}<2), 0$ lack of effect $(\mathrm{Di}=1), \downarrow$ less than twofold decrease

$(0.5<\mathrm{Di}<0.95), \downarrow \downarrow$ twothreefold decrease $(0.33 \leq \mathrm{Di} \leq 0.5), \downarrow \downarrow \downarrow$ more than threefold decrease $(\mathrm{Di}<0.33)$

\begin{tabular}{|c|c|c|c|c|c|c|}
\hline \multicolumn{2}{|c|}{ Growth regulators } & \multicolumn{4}{|c|}{ Effect on isoflavone accumulation ${ }^{\mathrm{a}}$} & \multirow{2}{*}{$\begin{array}{l}\text { Effect on } \\
\text { biomass } \\
\text { growth }\end{array}$} \\
\hline $\begin{array}{l}\text { Cytokinin } \\
\left(\mathrm{mg} \mathrm{l}^{-1}\right)\end{array}$ & $\begin{array}{l}\text { Auxin } \\
\left(\mathrm{mg} \mathrm{1}^{-1}\right)\end{array}$ & Aglycones & Glucosides & $\begin{array}{l}\text { Glucoside } \\
\text { esters }\end{array}$ & $\begin{array}{l}\text { Sum of } \\
\text { isoflavones }\end{array}$ & \\
\hline 5.0 & 0.5 & & & & & \\
\hline KN & - & $\mathrm{C}$ & $\mathrm{C}$ & $\mathrm{C}$ & $\mathrm{C}$ & $\mathrm{C}$ \\
\hline $\mathrm{KN}$ & 4-CРA & $\downarrow \downarrow \downarrow$ & $\downarrow \downarrow$ & $\downarrow \downarrow \downarrow$ & $\downarrow \downarrow$ & $\uparrow$ \\
\hline KN & 2,4-D & $\downarrow$ & $\downarrow$ & $\downarrow$ & $\downarrow$ & $\uparrow$ \\
\hline $\mathrm{KN}$ & $2,4,5-\mathrm{T}$ & $\downarrow \downarrow \downarrow$ & $\downarrow \downarrow$ & $\downarrow \downarrow \downarrow$ & $\downarrow \downarrow$ & $\uparrow \uparrow$ \\
\hline $\mathrm{KN}$ & IAA & $\downarrow$ & $\downarrow$ & $\downarrow \downarrow$ & $\downarrow$ & $\downarrow$ \\
\hline $\mathrm{KN}$ & IBA & $\downarrow$ & $\downarrow \downarrow$ & $\downarrow$ & $\downarrow$ & $\downarrow$ \\
\hline KN & IPA & 0 & $\downarrow$ & $\uparrow$ & $\downarrow$ & $\downarrow$ \\
\hline $\mathrm{KN}$ & NAA & $\downarrow$ & $\downarrow$ & $\downarrow \downarrow$ & $\downarrow$ & 0 \\
\hline $\mathrm{KN}$ & $\beta$-NOA & $\downarrow \downarrow \downarrow$ & $\uparrow$ & $\downarrow$ & $\uparrow$ & $\uparrow \uparrow \uparrow$ \\
\hline $\mathrm{KN}$ & PICL & $\downarrow \downarrow \downarrow$ & $\downarrow \downarrow$ & $\downarrow \downarrow$ & $\downarrow \downarrow$ & $\uparrow$ \\
\hline $\mathrm{KN}$ & TIBA & $\uparrow \uparrow$ & $\uparrow \uparrow \uparrow$ & $\downarrow$ & $\uparrow \uparrow \uparrow$ & $\uparrow \uparrow \uparrow$ \\
\hline 0.5 & 5.0 & & & & & \\
\hline KN & - & $\mathrm{C}$ & $\mathrm{C}$ & $\mathrm{C}$ & $\mathrm{C}$ & $\mathrm{C}$ \\
\hline $\mathrm{KN}$ & 4-CPA & $\uparrow \uparrow \uparrow$ & $\downarrow$ & $\downarrow$ & $\downarrow$ & $\downarrow \downarrow$ \\
\hline $\mathrm{KN}$ & $2,4-\mathrm{D}$ & $\uparrow \uparrow \uparrow$ & $\uparrow \uparrow \uparrow$ & $\uparrow \uparrow \uparrow$ & $\uparrow \uparrow \uparrow$ & $\uparrow$ \\
\hline $\mathrm{KN}$ & $2,4,5-\mathrm{T}$ & $\uparrow$ & $\downarrow$ & $\uparrow$ & 0 & $\downarrow \downarrow \downarrow$ \\
\hline $\mathrm{KN}$ & IAA & $\uparrow \uparrow$ & $\uparrow$ & $\uparrow$ & $\uparrow$ & \\
\hline $\mathrm{KN}$ & IBA & $\uparrow \uparrow \uparrow$ & $\uparrow$ & $\uparrow$ & $\uparrow$ & \\
\hline $\mathrm{KN}$ & IPA & $\uparrow \uparrow$ & $\downarrow \downarrow$ & $\downarrow$ & $\downarrow \downarrow$ & $\downarrow \downarrow \downarrow$ \\
\hline $\mathrm{KN}$ & NAA & $\uparrow \uparrow \uparrow$ & $\uparrow \uparrow$ & $\downarrow$ & $\uparrow \uparrow$ & 0 \\
\hline $\mathrm{KN}$ & $\beta$-NOA & $\uparrow$ & $\uparrow$ & $\uparrow$ & $\uparrow$ & $\downarrow \downarrow$ \\
\hline $\mathrm{KN}$ & PICL & $\downarrow$ & $\downarrow$ & $\downarrow$ & $\downarrow$ & $\downarrow \downarrow \downarrow$ \\
\hline $\mathrm{KN}$ & TIBA & $\uparrow \uparrow \uparrow$ & $\uparrow$ & $\uparrow \uparrow \uparrow$ & $\uparrow$ & $\uparrow$ \\
\hline 0.5 & 5.0 & & & & & \\
\hline- & 2,4-D & $\mathrm{C}$ & $\mathrm{C}$ & $\mathrm{C}$ & $\mathrm{C}$ & $\mathrm{C}$ \\
\hline BAP & 2,4-D & 0 & $\uparrow$ & $\uparrow \uparrow$ & $\uparrow$ & 0 \\
\hline 4-CPPU & 2,4-D & $\downarrow \downarrow$ & $\uparrow \uparrow$ & $\uparrow$ & $\uparrow \uparrow$ & 0 \\
\hline 1,3-DPU & 2,4-D & $\downarrow \downarrow \downarrow$ & $\downarrow \downarrow$ & $\downarrow$ & $\downarrow \downarrow$ & 0 \\
\hline 2iP & 2,4-D & $\downarrow$ & $\uparrow$ & 0 & $\uparrow$ & $\uparrow$ \\
\hline KIN & 2,4-D & $\uparrow$ & $\uparrow \uparrow \uparrow$ & $\uparrow \uparrow \uparrow$ & $\uparrow \uparrow \uparrow$ & $\uparrow \uparrow$ \\
\hline TDZ & 2,4-D & $\downarrow$ & $\uparrow \uparrow$ & $\uparrow$ & $\uparrow \uparrow$ & 0 \\
\hline ZEA & 2,4-D & $\downarrow \downarrow \downarrow$ & $\downarrow$ & $\uparrow$ & $\downarrow$ & $\uparrow$ \\
\hline 5.0 & 0.5 & & & & & \\
\hline- & 2,4-D & $\mathrm{C}$ & $\mathrm{C}$ & C & $\mathrm{C}$ & $\mathrm{C}$ \\
\hline BAP & 2,4-D & $\uparrow$ & $\uparrow \uparrow$ & $\uparrow$ & $\uparrow$ & $\uparrow$ \\
\hline 4-CPPU & 2,4-D & $\uparrow$ & $\uparrow$ & $\downarrow$ & $\uparrow$ & $\uparrow \uparrow \uparrow$ \\
\hline 1,3-DPU & 2,4-D & $\downarrow \downarrow$ & $\downarrow \downarrow$ & $\downarrow \downarrow$ & $\downarrow \downarrow$ & $\uparrow$ \\
\hline 2iP & 2,4-D & $\uparrow$ & $\uparrow$ & $\downarrow$ & $\uparrow$ & $\uparrow \uparrow \uparrow$ \\
\hline KIN & 2,4-D & $\uparrow$ & $\uparrow$ & $\uparrow$ & $\uparrow$ & $\uparrow$ \\
\hline TDZ & 2,4-D & $\uparrow$ & $\uparrow$ & $\downarrow$ & $\uparrow$ & $\uparrow$ \\
\hline ZEA & 2,4-D & $\downarrow$ & $\downarrow$ & $\downarrow$ & $\downarrow$ & $\downarrow$ \\
\hline
\end{tabular}

The collected biomasses were freeze-dried using LYOVAC GT2 apparatus (Finn-Aqua Santasolo-Sohlberg, Tuusula, Finland). Extracts preparation, as well as qualitative and quantitative HPLC analyses of isoflavones were performed, following the procedures described previously (Łuczkiewicz and Głód 2003). 

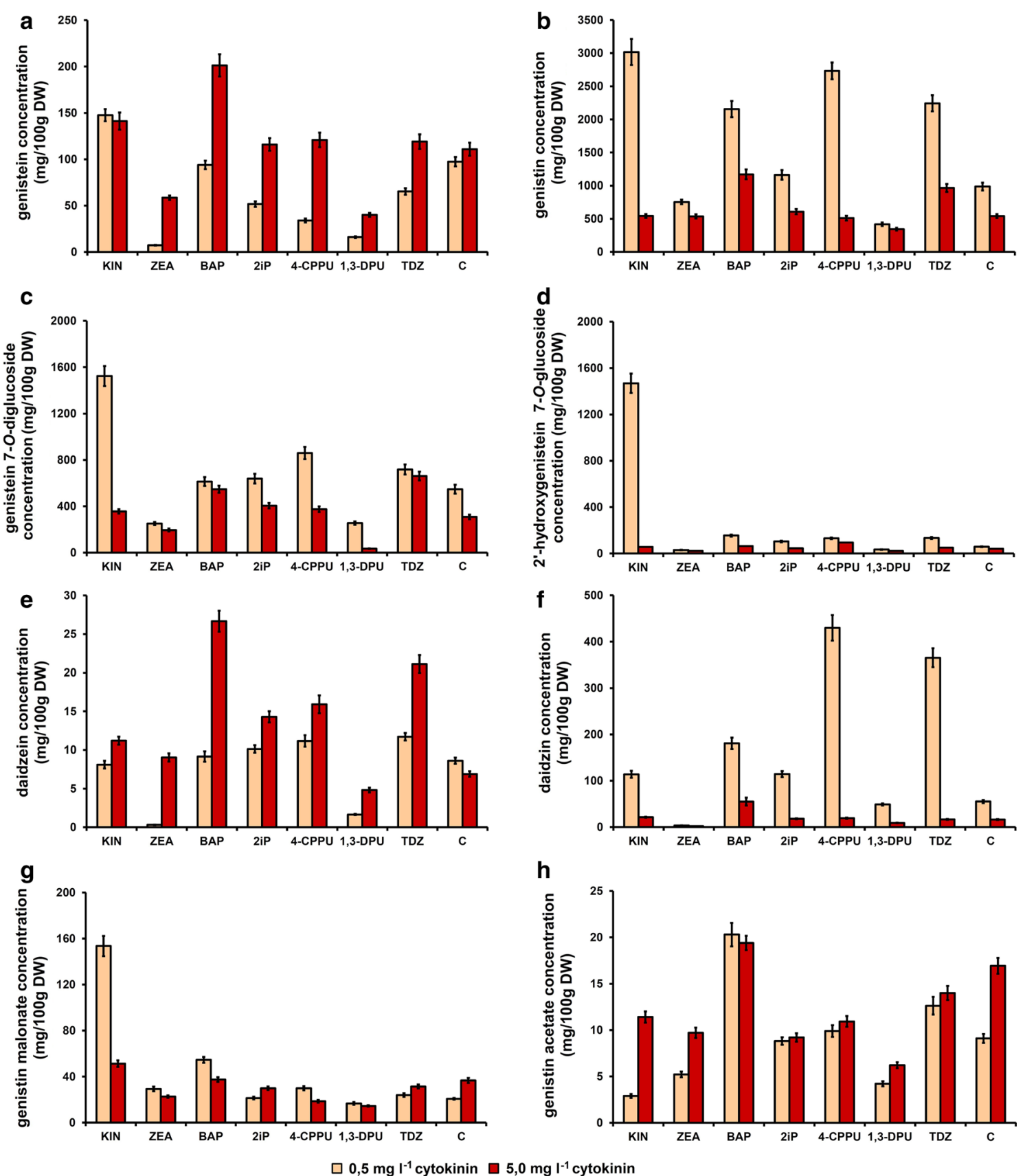

Fig. 2 The effect of various cytokinins and their concentrations on the accumulation of genistein (a), genistin (b), genistein 7- $O$ diglucoside (c), 2'-hydroxygenistein 7-O-glucoside (d), daidzein (e),

daidzin (f), genistin malonate (g) and genistin acetate (h) in Genista tinctoria callus. Values represent the mean \pm SD of 15 replicates

\section{Statistical analyses}

The quantitative effect of PGRs on biomass growth and isoflavone accumulation in G. tinctoria calli was evaluated using the analysis of dynamics, by introducing dynamics index (Di) defined by the formula: $\mathrm{Di}=\mathrm{x}_{\mathrm{n}} / \mathrm{x}_{0}$, where $\mathrm{x}_{0}$ denotes an average of a series of measurements of the concentration of an investigated isoflavone (or the $\mathrm{Gi}$ value) in the control experiment and $x_{n}$ is an average of the

same parameter in subsequent experiments. The Di properties are as follows: (1) it is an abstract number, (2) it is a non-negative number $(\mathrm{Di} \leq 1)$, (3) it equals one if the values of the analysed parameters are in both cases equal $\left(x_{n}=x_{0}\right),(4)$ it is larger than one ( $\left.\mathrm{Di}>1\right)$ when the value of the parameter in the nth experiment is larger than the value in the control experiment and (5) it is smaller than one $(\mathrm{Di}<1)$ when the value of the parameter in the nth experiment is smaller than the value in the control group. It 

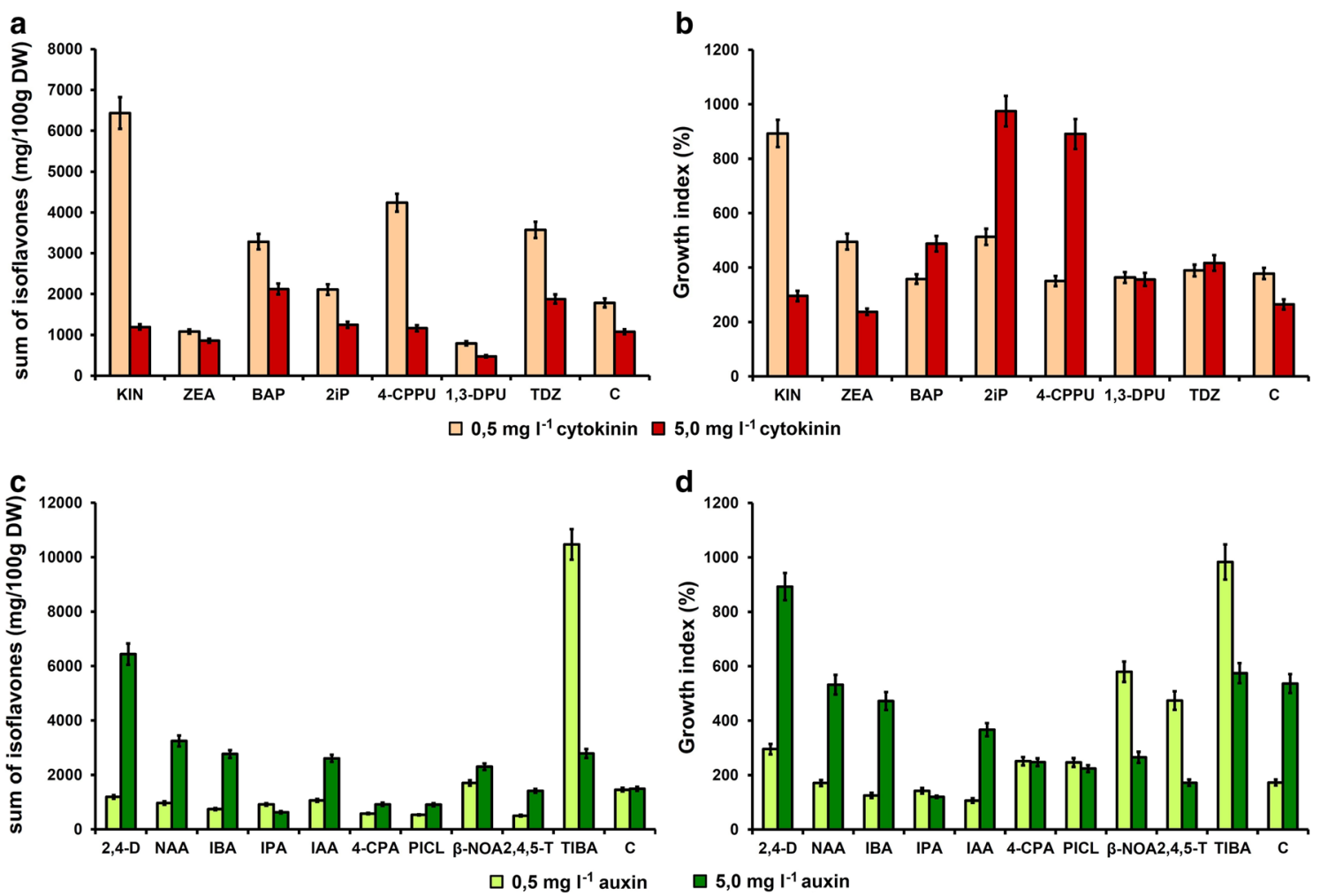

Fig. 3 The effect of various cytokinins, auxins and their concentrations on the total accumulation of isoflavones $(\mathbf{a}, \mathbf{c})$ and the value of the growth index $(\mathbf{b}, \mathbf{d})$ of Genista tinctoria callus. Growth indices were calculated using the formula: $\mathrm{Gi}=\left[\left(\mathrm{G}_{1}-\mathrm{G}_{0}\right) / \mathrm{G}_{0}\right] \times 100$ where

was assumed that the value of $\mathrm{Di}=1 \pm 0.05$ denoted no change in the evaluated parameter, $\mathrm{Di}<0.95$ indicated inhibitory effect of a given experimental modification and Di $>1.05$ indicated a stimulatory effect (Table 1). The adopted range $(-0.05 \div 0.05)$ followed from the assumed $5 \%$ level of significance in comparing results of the various experiments.

Further statistical analyses were conducted to establish linear correlation coefficients $\left(\mathrm{r}_{\mathrm{xy}}\right)$ between concentrations of the analysed isoflavones and Gi values of G. tinctoria calli grown using different combinations of PGRs. As presented in Table 2, the resulting $\mathrm{r}_{\mathrm{xy}}$ values were calculated for four sets of media modifications, with variable auxins or variable cytokinins added at 0.5 or $5.0 \mathrm{mg} \mathrm{l}^{-1}$. The scale usually adopted in statistical analyses was applied to evaluate the correlation between the aforementioned parameters: $r_{x y}=0$-variables not correlated, $0<\mathrm{r}_{\mathrm{xy}}<0.1$-minute correlation, $0.1<\mathrm{r}_{\mathrm{xy}}<0.3$ - poor correlation, $\quad 0.3<\mathrm{r}_{\mathrm{xy}}<0.5$-average correlation, $0.5<\mathrm{r}_{\mathrm{xy}}<0.7$-high correlation, $0.7<\mathrm{r}_{\mathrm{xy}}<0.9$-very high correlation, $0.9<\mathrm{r}_{\mathrm{xy}}<1$-almost full correlation. Positive linear correlation coefficients indicate positive correlation between the analysed parameters and negative coefficients indicate reverse correlation. All statistical
$\mathrm{Gi}$ is the growth index, $\mathrm{G}_{1}$ is the callus fresh weight after a 4-week cultivation period and $\mathrm{G}_{0}$ is the fresh weight of the inoculum. Values represent the mean \pm SD of 15 replicates

Table 2 The values of linear correlation coefficient $\left(r_{x y}\right)$ between the concentrations of isoflavones and the growth indices of $G$. tinctoria callus grown on $\mathrm{SH}$ media modified with different concentrations of auxins and cytokinins

\begin{tabular}{lr}
\hline Growth regulator combination & \multicolumn{1}{c}{$\mathrm{r}_{\mathrm{xy}}$} \\
\hline $0.5 \mathrm{mg} \mathrm{l^{-1 }} \mathrm{KIN}+5.0 \mathrm{mg} \mathrm{l}^{-1}$ auxins & -0.31 \\
$5.0 \mathrm{mg} \mathrm{l}^{-1} \mathrm{KIN}+0.5 \mathrm{mg} \mathrm{l}^{-1}$ auxins & -0.39 \\
$0.5 \mathrm{mg} \mathrm{l}^{-1} 2.4-\mathrm{D}+5.0 \mathrm{mg} \mathrm{l}^{-1}$ cytokinins & 0.13 \\
$5.0 \mathrm{mg} \mathrm{l}^{-1} 2.4-\mathrm{D}+0.5 \mathrm{mg} \mathrm{l}^{-1}$ cytokinins & 0.61 \\
\hline
\end{tabular}

analyses were carried out using STATISTICA software (StatSoft, Tulsa, US-OK).

\section{Results}

The effect of variable auxin on biomass growth and isoflavone production

The experiment included 10 natural and synthetic auxins of varying strength, commonly used to grow higher plants in vitro. The PGRs used showed considerable effect on 
biomass growth and the accumulation of isoflavones in $G$. tinctoria callus. The observed relations were directly caused by the type of phytohormone used and its concentration in the growth medium (Figs 1, 3; Table 1). It was determined that auxins added to SH media in low concentration of $0.5 \mathrm{mg} 1^{-1}$ generally inhibited the production of isoflavones (Fig. 1). This was true for the total isoflavones and, selectively, for aglycones, glucosides and esters (Fig. 3, Table 1). Exceptions to this rule are $\beta$-NOA and TIBA, which at $0.5 \mathrm{mg} 1^{-1}$ conditioned an increase of aglycones and isoflavonoid glucosides in the investigated calli, as well as IPA which selectively stimulated the production of genistin malonate (Fig. 1).

A comparison of the $\mathrm{Gi}$ values for biomasses grown on media supplemented with $0.5 \mathrm{mg}^{-1}$ auxins showed that except for IAA, IBA and IPA, the tested compounds stimulated the growth of G. tinctoria calli (Fig. 3, Table 1). In this series of experiments, the fastest growth ( $\mathrm{Gi}=983.07 \%$-Fig. 3) and the highest total isoflavone concentration $(10,474.23 \mathrm{mg} / 100 \mathrm{~g}$ dry weight-Fig. 3) were achieved on the medium supplemented with $0.5 \mathrm{mg}^{-1}$ TIBA and $5.0 \mathrm{mg}^{-1} \mathrm{KIN}$. Statistical analysis showed average inverse relationship between the total concentration of isoflavones and the growth ratios of calli cultivated on media supplemented with $0.5 \mathrm{mg} \mathrm{l}^{-1}$ auxins (Table 2).

In contrast with the above described experiments, high concentration of auxins $\left(5.0 \mathrm{mg}^{-1}\right)$ in the experimental media was, in the case of most tested phytohormones, associated with increased accumulation of isoflavones in $G$. tinctoria calli (Fig. 1). It was true for aglycones, glucosides, glucoside esters and total isoflavones (media modified with 2,4-D, NAA, IBA, TIBA, IAA and $\beta$-NOATable 1). Moreover, the auxins used, except for 2,4-D and TIBA, inhibited biomass growth when supplemented at $5.0 \mathrm{mg} \mathrm{l}^{-1}$ (Fig. 3; Table 1). Like in the experiments with media containing $0.5 \mathrm{mg} \mathrm{l}^{-1}$ auxins, an average inverse correlation was demonstrated between biomass growth and isoflavone production (Table 2).

The effect of variable cytokinin on biomass growth and isoflavone production

The experiment included 7 compounds classified as natural or synthetic cytokinins. As in the case of auxin studies, it was observed that cytokinins significantly influenced cell growth and isoflavone production in G. tinctoria calli (Figs. 2, 3; Table 1). The observed effects depended on the type of PGR used, as well as its concentration in the growth medium.

It was determined that except for ZEA and 1,3-DPU, cytokinins at $0.5 \mathrm{mg}^{-1}$ significantly stimulated the production of isoflavonoid glucosides and glucoside esters
(Table 1). In these experimental conditions, a significant increase of the total content of the investigated secondary metabolites in G. tinctoria calli was also noted (Fig. 3; Table 1). On the other hand, experimental media supplemented with $0.5 \mathrm{mg}^{-1}$ of the selected cytokinins (except KIN) inhibited the accumulation of aglycones (Fig. 2; Table 1).

Comparative analysis of the $\mathrm{Gi}$ values of the calli grown on media supplemented with low concentrations of various cytokinins showed that these PGRs either did not significantly influence biomass growth (1,3-DPU, 4-CPPU, TDZ, BAP) or had a stimulatory effect (KIN, 2iP, ZEATable 1). In the described series of experiments, the fastest growth $(\mathrm{Gi}=892.46 \%$-Fig. 3$)$ and the highest isoflavone content $(6,436.26 \mathrm{mg} / 100 \mathrm{~g}$ dry weight-Fig. 2) were obtained on the medium supplemented with $0.5 \mathrm{mg} \mathrm{l}^{-1}$ $\mathrm{KIN}$ and $5.0 \mathrm{mg} \mathrm{l}^{-1}$ 2,4-D. Statistical analysis showed a poor, positive correlation between the growth ratios and accumulation of isoflavones in $G$. tinctoria cultures (Table 2).

Cytokinins supplemented at $5.0 \mathrm{mg} \mathrm{l}^{-1}$, except for 1,3DPU and ZEA, stimulated the accumulation of isoflavone glucosides in a similar way as when they were applied at $0.5 \mathrm{mg}^{-1}$. This was also true with respect to total isoflavone content in G. tinctoria calli (Figs. 2, 3; Table 1). The observed increase in isoflavones production under the influence of various cytokinins applied at $5.0 \mathrm{mg} \mathrm{l}^{-1}$ was, however, significantly weaker (maximum concentration of $2,122.01 \mathrm{mg} / 100 \mathrm{~g}$ dry weight obtained for $5.0 \mathrm{mg} \mathrm{l}^{-1}$ BAP-Fig. 2) in comparison to medium modifications containing only $0.5 \mathrm{mg}^{-1}$ of these phytohormones. Unlike experimental variants including $0.5 \mathrm{mg} \mathrm{l}^{-1}$ cytokinin supplementation, higher concentrations of these PGRs stimulated the production of isoflavonoid aglycones in the biomasses. The growth media applied, except for those supplemented with KIN and BAP, also hampered the accumulation of glucoside esters (Fig. 2; Table 1). In this case a high, positive correlation was observed between the callus growth and isoflavone accumulation (Table 2).

\section{Discussion}

Numerous reports indicate a direct influence of PGRs on the biosynthesis of phenolic secondary metabolites in plant in vitro cultures, with the examples including medicinally relevant antraquinones (Stalman et al. 2003; Smetanska 2008), naphthodianthrones, phloroglucinols (Coste et al. 2011), phenolic acids (Szopa et al. 2013; Szopa and Ekiert 2014) and anthocyanins (Matkowski 2008, Smetanska 2008). Phytohormones may either stimulate or inhibit the production of active compounds in cultivated biomasses which, unfortunately, is not always correlated with the 
induction or inhibition of cell growth (Stalman et al. 2003; Collin 2001). As a result, it is sometimes rather hard to select experimental media providing both satisfactory biomass yield and high concentration of the desired compounds. This problem is usually resolved by using two stage production systems in which cell growth and the biosynthesis of secondary metabolites are achieved in two different experimental media (Collin 2001; Chattopadhyay et al. 2002). Such approach, however, is relatively costly and poses certain technological problems. Therefore, it seems essential to select the right experimental media, also in terms of PGRs, to achieve intensive biomass growth and secondary metabolite production in a single batch culture.

In the presented experiment, 17 auxins and cytokinins were tested for the first time in a single growth model (Figs. 1, 2 and 3; Table 1). Apart from the phytohormones commonly applied to cell cultures of legume plants, such as BAP, 2,4-D, IAA, KIN, NAA and ZEA (Fedoreyev et al. 2000; Luo et al. 2003; Matkowski 2004; Vaishnav et al. 2006; Tůmová et al. 2007; Shinde et al. 2010; Pu et al. 2013), the study included PGRs which so far have not been widely examined with respect to their influence on isoflavone metabolism. Considering that the biosynthesis of isoflavonoids under in vitro conditions depends not only on PGR type, but also on its amount in the growth medium (Fedoreyev et al. 2000; Vaishnav et al. 2006; Tůmová et al. 2007; Goyal and Ramawat 2008; Shinde et al. 2010), the particular auxins and cytokinins were supplemented in two extreme concentrations of 0.5 and $5.0 \mathrm{mg}^{-1}$. Due to the fact that $G$. tinctoria cultures died rapidly on the hormone-free medium (data not shown), and consequently could not serve as an optimal control group (in such a case, the application of any PGR would always seemingly stimulate cell growth, making valid conclusions about the activity of the respective auxins and cytokinins impossible), the reference samples were calli grown on SH medium supplemented solely with KIN or 2,4D. As indicated in previous reports, both PGRs or their combinations were successfully used for the initiation and maintenance of callus cultures of Genista plants (Łuczkiewicz and Głód 2003; Tůmová et al. 2007; Tůmová and Tůma 2011), as well as a representative of the closely related genus Cytisus (Lucchesini et al. 2010).

The presented work is the first comprehensive report describing the selective effect of auxins and cytokinins on the accumulation of aglycones, glycosides and isoflavonoid esters. The comparable analysis included derivatives of genistein and daidzein, i.e. the main metabolites in the 16-component isoflavone set present in the investigated species (Łuczkiewicz et al. 2004). Moreover, in comparison to some other research (Vaishnav et al. 2006; Tůmová et al. 2007), the presented series of experiments was conducted with the use of the cell line which is particularly productive with respect to isoflavones (total content up to ca. $6.5 \%$ dry weight—Łuczkiewicz and Głód 2003), and therefore more suitable for studying relationships between primary and secondary metabolism (Stalman et al. 2003).

In general, the results of the present work confirmed literature reports regarding the difficulties in developing media which would condition both intensive biomass growth and high accumulation of the desired metabolites. The problem arises from the fact that the pathways of primary and secondary metabolism compete for precursors, which is particularly noticeable in the case of high productive cell lines (Collin 2001; Stalman et al. 2003). In this respect, auxins deserve special attention due to their regulatory role between the above metabolic branches. The use of auxins is often associated with decreased cell differentiation and inhibition of secondary metabolism (Collin 2001). 2,4-D, for example, was shown to completely hamper the production of anthocyanins and antraquinones in the model systems of Daucus carota and Morinda citrifolia, respectively (Ozeki 1996; Stalman et al. 2003). As far as the isoflavonoids are concerned, literature data do not indicate that their accumulation in cell cultures is negatively influenced by auxin supplementation. In fact, several high-productive cell lines, including those of Glycine max, $P$. corylifolia and M. amurensis (isoflavone content up to ca. 7, 1.8 and $1.3 \%$ dry weight, respectively), were successfully maintained on 2,4-D-enriched media (Fedoreyev et al. 2000; Federici et al. 2003; Shinde et al. 2010). However, these reports provide limited information concerning the relative potency of 2,4-D in comparison to other auxins, as well as the interactions between the respective PGRs.

In the presented series of experiments 2,4-D, as well as some other compounds including NAA, IBA and IAA, proved to stimulate isoflavone production in G. tinctoria calli at $5.0 \mathrm{mg}^{-1}$ when applied jointly with $0.5 \mathrm{mg} \mathrm{l}^{-1}$ cytokinin-Figs. 1, 3; Table 1). In this regard 2,4-D showed greater potency in comparison to NAA, IAA and IBA. As far as NAA is concerned, this observation is in agreement with previous reports (Fedoreyev et al. 2000; Vaishnav et al. 2006; Shinde et al. 2010), however, similar relationship was not reported with respect to IAA and IBA (Vaishnav et al. 2006; Shinde et al. 2010). As in the present work, auxins in the above mentioned experiments were applied together with cytokinin.

Among the tested auxins 2,4-D deserves special attention because of its growth-promoting properties, demonstrated in this study. This PGR, aside from TIBA, was the only auxin which at $5.0 \mathrm{mg} \mathrm{l}^{-1}$ stimulated the growth of G. tinctoria culture ( $\mathrm{Gi}=892.46 \%$-Fig. 3 ). Moreover, the callus grown on the medium supplemented with $5.0 \mathrm{mg}^{-1}$ 2,4-D and $0.5 \mathrm{mg} \mathrm{l}^{-1} \mathrm{KIN}$ accumulated the highest amounts of isoflavones in the whole experiment $(6,436.26 \mathrm{mg} / 100 \mathrm{~g}$ dry weight—Fig. 1). 
Of all the auxins tested, the most surprising results were obtained using the media supplemented with TIBA. Unlike other PGRs in this class, TIBA proved to be the strongest stimulant of isoflavone production in G. tinctoria callus when used at $0.5 \mathrm{mg} \mathrm{l}^{-1}$ together with $5.0 \mathrm{mg} \mathrm{l}^{-1} \mathrm{KIN}$ $(10,474.23 \mathrm{mg}$ total isoflavones per $100 \mathrm{~g}$ dry weightFig. 3). As in the case of 2,4-D, the effect on secondary metabolism was correlated with intensive biomass growth $(\mathrm{Gi}=983.07$-Fig. 3). The interpretation of these results, especially in terms of TIBA-triggered induction of isoflavone biosynthesis, seems rather difficult at the current stage of research. As far as the authors are concerned, there are no reports on the effect of TIBA on the biosynthesis of bioflavonoids in cell cultures of higher plants which could serve for comparative purposes. However, the result has implications for studies aimed at establishing large-scale systems for the production of isoflavones. As described in recent work by the authors (Luczkiewicz and Kokotkiewicz 2012), G. tinctoria callus grown on the above medium proved suitable for establishing high-productive suspension cultures (isoflavone content up to ca. $11 \%$ dry weight), which can be further used for bioreactor studies.

The second part of the presented research was devoted to the evaluation of the influence of different cytokinins, applied in variable concentrations, on biomass growth and isoflavones accumulation in $G$. tinctoria calli. As in the case of auxins, the observed effects depended largely on the type of PGR used. In general, the experiment confirmed that the simultaneous use of auxin and cytokinin often stimulates the production of secondary metabolites (Goyal and Ramawat 2008). Of all the tested cytokinins, KIN at $0.5 \mathrm{mg} \mathrm{l}^{-1}$ exerted the strongest stimulatory effect on isoflavone production (Figs. 2, 3; Table 1). Given the lack of data on the role of this PGR in isoflavone biosynthesis, it is difficult to assess the mechanism of the described phenomenon. The obtained results, however, confirmed other reports that the combination of 2,4D and KIN, at certain concentration ranges, exerts stimulatory effect on isoflavonoid biosynthesis in G. tinctoria callus (Tůmová et al. 2007; Tůmová and Tůma 2011). Isoflavone concentration achieved in the present work (ca. $6.5 \%$ dry weight) was also higher in comparison to previous studies on cell cultures of the examined species (up to ca. $1.1 \%$ dry weight - data for callus grown without UV irradiation [Tůmová and Tůma 2011]).

6- $(\gamma, \gamma$-Dimethylallylamino)purine (2-isopentenyladenine), as well as 3 phenylurea derivatives (4-CPPU, 1,3DPU and TDZ) included in the present work, have so far not been extensively examined with respect to their influence on isoflavone metabolism under in vitro conditions. Goyal and Ramawat (2008) reported that 2iP stimulated isoflavone production in $P$. tuberosa callus when applied alone, together with 2,4,5-T or jointly with 2,4,5-T + KIN (Goyal and
Ramawat 2008). Similarly, Udomsuk et al. (2009, 2012) described the stimulatory effect of TDZ, used as the sole PGR or conjointly with NAA + BAP, on isoflavone accumulation in callus cultures of Pueraria candollei var. mirifica. The present study confirmed these observations only with respect to TDZ (Fig. 2), however, the results of the reports are not directly comparable due to different PGR compositions and plant models used. Beside TDZ, significant increase in isoflavonoid content was recorded for 4-CPPU $(4,238.59 \mathrm{mg} / 100 \mathrm{~g}$ dry weight-Fig. 3). Interestingly, at $0.5 \mathrm{mg}^{-1}$ both PGRs selectively stimulated the accumulation of isoflavone glucosides (Fig. 2).

Although the use of cytokinins is usually associated with increased cytodifferentiation, some studies indicate their stimulatory effect on the growth of unorganized cultures. Among the adenine derivatives included in this work, such effect was found for $2 \mathrm{iP}(\mathrm{Gi}=974.4 \%$-Fig. 3, Table 1) and, to a lesser extent, BAP (both PGRs applied at $5.0 \mathrm{mg} \mathrm{l}^{-1}$ ). The results are in agreement with other reports concerning cell cultures of legume plants. In the study by Goyal and Ramawat (2008), 2iP at $5.0 \mathrm{mg}^{-1}$ significantly stimulated the growth of $P$. tuberosa callus when supplemented to the medium jointly with $2,4,5-\mathrm{T}$ and KIN. However, the observed effect (ca. $20 \%$ increase in dry weight) was less prominent than in the present work (nearly fourfold increase in Gi value-Fig. 3). In another experiment, conducted on in vitro cultures of Pueraria lobata, BAP clearly induced callus growth when introduced to NAA- or 2,4-D-containing media as a second PGR (Matkowski 2004).

As far as phenylurea-type cytokinins are concerned, the present work determined noticeable stimulatory effect on biomass growth only for 4-CPPU, applied at $5.0 \mathrm{mg} \mathrm{l}^{-1}$ (Fig. 3). So far, there is limited data on the use of this class of PGRs in cell cultures of legume plants. In the work concerning shoot organogenesis in common and faba beans, Mohamed et al. (1992) found that both TDZ and 4-CPPU promoted callus formation in concentrations exceeding $0.5 \mathrm{mg}^{-1}$. More recently, Udomsuk et al. (2009) recorded high callus induction rates in Pueraria candollei var. mirifica explants cultivated on the media supplemented with TDZ in the range $0.1-1.5 \mathrm{mg}^{-1}$, but no growth rates were provided. In the experiments with in vitro cultures of the South-African legumes of the genus Cyclopia, 4-CPPU-supplemented media provided the fastest callus growth, with Gi values exceeding 1,200\% for $C$. subternata (Kokotkiewicz et al. 2009). The medium of the same composition also proved suitable for establishing isoflavone-producing suspension cultures of the above species (Kokotkiewicz et al. 2013).

The statistical analysis of the obtained results showed the relationships between callus growth and accumulation of isoflavones for the respective groups of investigated 
phytohormones. Depending on PGRs concentrations applied, poor to high positive correlation was found for cytokinins, whereas average negative correlation was established within the auxin group (Table 2). Although the observed correlations do not necessarily imply causal relationship, these results generally reflect the typical pattern of activity of exogenous PGRs under in vitro conditions (i.e. increase in primary metabolism and inhibition of secondary metabolism for auxins and, otherwise, stimulatory effect of cytokinins on secondary metabolite pathways). However, as discussed above, these conclusions are true only for the PRG groups as a whole, but not necessarily for the particular auxins and cytokinins.

\section{Conclusions}

To sum up, the presented work determined the influence of different compositions of auxins and cytokinins on biomass growth and the accumulation of isoflavones in callus cultures of $G$. tinctoria. This is the first such comprehensive report on the effects of exogenous PGRs on isoflavonoid metabolism in cell cultures of legume plants, including 17 phytohormones in 36 different combinations. Despite obvious limitations (lack of data on PGRs activity at genomic, transcriptomic and proteomic levels) the current work provides good basis for further experiments on the influence of auxins and cytokinins on isoflavone biosynthesis. Most importantly, the conducted experiments included a large number of PGRs, evaluated for their activity in a single growth model. Therefore, the study provides more reliable information on the relative potency of the respective phytohormones than the compilation of data from several experiments, which are often hardly comparable because of the use of different plant models, explant sources, culture conditions and PGR concentration ranges. Moreover, G. tinctoria in vitro culture used in this research produces large quantities of isoflavones in the form of aglycones, glucosides and glucoside esters, which make it suitable for studies on the relationships between different branches of isoflavonoid biosynthesis, as well as interactions between primary and secondary metabolism. Apart from the above, G. tinctoria cell cultures can be potentially used for the production of medicinally-relevant isoflavones. With isoflavone content exceeding $10 \%$ dry weight, callus cultures of this species maintained on $\mathrm{SH}$ medium supplemented with $0.5 \mathrm{mg} \mathrm{l}^{-1}$ TIBA and $5.0 \mathrm{mg} \mathrm{l}^{-1} \mathrm{KIN}$ are so far the richest source of isoflavonoids among in vitro cultures of higher plants. In the previous research (Luczkiewicz and Kokotkiewicz 2012), the authors have already succeeded in establishing high-productive suspension cultures of $G$. tinctoria using liquid medium with the same PGR composition. However, further scale-up studies in bioreactors are required to establish an efficient system for isoflavone production.

Open Access This article is distributed under the terms of the Creative Commons Attribution License which permits any use, distribution, and reproduction in any medium, provided the original author(s) and the source are credited.

\section{References}

Behloul N, Wu G (2013) Genistein: a promising therapeutic agent for obesity and diabetes treatment. Eur J Pharmacol 698:31-38. doi:10.1016/j.ejphar.2012.11.013

Chattopadhyay S, Farkya S, Srivastava AK, Bisaria VS (2002) Bioprocess considerations for production of secondary metabolites by plant cell suspension cultures. Biotechnol Bioprocess Eng 7:138-149. doi:10.1007/BF02932911

Collin HA (2001) Secondary product formation in plant tissue cultures. Plant Growth Regul 34:119-134. doi:10.1023/A:1013374417961

Coste A, Vlase L, Halmagyi A, Deliu C, Coldea G (2011) Effects of plant growth regulators and elicitors on production of secondary metabolites in shoot cultures of Hypericum hirsutum and Hypericum maculatum. Plant Cell Tissue Organ Cult 106:279-288. doi:10.1007/s11240-011-9919-5

Du H, Huang Y, Tang Y (2010) Genetic and metabolic engineering of isoflavonoid biosynthesis. Appl Microbiol Biotechnol 86:1293-1312. doi:10.1007/s00253-010-2512-8

Engelmann NJ, Reppert A, Yousef G, Rogers RB, Lila MA (2009) In vitro production of radiolabeled red clover (Trifolium pratense) isoflavones. Plant Cell Tissue Organ Cult 98:147-156. doi:10. 1007/s11240-009-9547-5

Federici E, Touché A, Choquart S, Avanti O, Fay L, Offord E, Courtois D (2003) High isoflavone content and estrogenic activity of 25 year-old Glycine max tissue cultures. Phytochemistry 64:717-724. doi:10.1016/S0031-9422(03)00379-0

Fedoreyev SA, Pokushalova TV, Veselova MV, Glebko LI, Kulesh NI, Muzarok TI, Seletskaya LD, Bulgakov VP, Zhuravlev YN (2000) Isoflavonoid production by callus cultures of Maackia amurensis. Fitoterapia 71:365-372. doi:10.1016/S0367-326X(00)00129-5

Fedoreyev SA, Bulgakov VP, Grishchenko OV, Veselova MV, Krivoschekova OE, Kulesh NI, Denisenko VA, Tchernoded GK, Zhuravlev YN (2008) Isoflavonoid composition of a callus culture of the relict tree Maackia amurensis Rupr. et Maxim. J Agric Food Chem 56:7023-7031. doi:10.1021/jf801227q

Fritz H, Seely D, Flower G, Skidmore B, Fernandes R, Vadeboncoeur S, Kennedy D, Cooley K, Wong R, Sagar S, Sabri E, Fergusson D (2013) Soy, red clover, and isoflavones and breast cancer: a systematic review. PLoS ONE 8:e81968. doi:10.1371/journal.pone.0081968

Goyal S, Ramawat KG (2008) Synergistic effect of morphactin on cytokinin-induced production of isoflavonoids in cell cultures of Pueraria tuberosa (Roxb. ex. Willd.) DC. Plant Growth Regul 55:175-181. doi:10.1007/s10725-008-9271-x

Kašparová M, Siatka T, Klimešová V, Dušek J (2012) New synthetic pyridine derivate as potential elicitor in production of isoflavonoids and flavonoids in Trifolium pratense L. suspension culture. Sci World. doi:10.1100/2012/746412

Kokotkiewicz A, Wnuk M, Bucinski A, Luczkiewicz M (2009) In vitro cultures of Cyclopia plants (honeybush) as a source of bioactive xanthones and flavanones. Z Naturforsch 64c:533540

Kokotkiewicz A, Luczkiewicz M, Kowalski W, Badura A, Piekus N, Bucinski A (2013) Isoflavone production in Cyclopia subternata Vogel (honeybush) suspension cultures grown in shake flasks 
and stirred-tank bioreactor. Appl Microbiol Biotechnol 97:8467-8477. doi:10.1007/s00253-013-5099-z

Lei Z, Chen F, Watson BS, Nagaraj S, Elmer AM, Dixon RA, Sumner LW (2010) Comparative proteomics of yeast-elicited Medicago truncatula cell suspensions reveals induction of isoflavonoid biosynthesis and cell wall modifications. J Proteome Res 9:6220-6231. doi:10.1021/pr100439k

Li Y, Kong D, Bao B, Ahmad A, Sarkar FH (2011) Induction of cancer cell death by isoflavone: the role of multiple signalling pathways. Nutrients 3:877-896. doi:10.3390/nu3100877

Lucchesini M, Bertoli A, Mensuali-Sodi A, Cappelli E, Noccioli C, Luciardi L, Pistelli L (2010) Cytisus aeolicus Guss. ex Lindl. in vitro cultures and genistin production. Cent Eur J Biol 5:111-120. doi:10.2478/s11535-009-0067-4

Luczkiewicz MT (2008) Research into isoflavonoid phyto-oestrogens in plant cell cultures. In: Ramawat KG, Mérillon (eds) Bioactive molecules and medicinal plants. Springer, Berlin-Heidelberg, pp 55-84. doi: 10.1007/978-3-540-74603-4_3

Łuczkiewicz M, Głód D (2003) Callus cultures of Genista plantsin vitro material producing high amounts of isoflavones of phytoestrogenic activity. Plant Sci 165:1101-1108. doi:10.1016/ S0168-9452(03)00305-4

Luczkiewicz M, Kokotkiewicz A (2012) Elicitation and permeabilization affect the accumulation and storage profile of phytoestrogens in high productive suspension cultures of Genista tinctoria. Acta Physiol Plant 34:1-16. doi:10.1007/s11738-011-0799-4

Łuczkiewicz M, Głód D, Bączek T, Buciński A (2004) LC-DAD UV and LC-MS for the analysis of isoflavones and flavones from in vitro and in vivo biomass of Genista tinctoria L. Chromatographia 60:179-185. doi:10.1365/s10337-004-0357-y

Luo JP, Wu LF, Jiang ST (2003) Callus formation and its isoflavonoid accumulation in Maackia amurensis. Zhongguo Zhong Yao Za Zhi 28:1138-1141

Luo JP, Luo K, Chen XY, Jiang ST (2004) Optimization for isoflavone production in Maackia amurensis suspension cells based on neural networks and accelerating genetic algorithm. Sheng Wu Gong Cheng Xue Bao 20:759-763

Luo K, Zha X-Q, Luo J-P (2007) Structure kinetic model of Maackia amurensis cells in suspension culture. Sheng Wu Gong Cheng Xue Bao 23:657-661

Mahmoud AM, Yang W, Bosland MC (2014) Soy isoflavones and prostate cancer: a review of molecular mechanisms. J Steroid Biochem Mol Biol 140:116-132. doi:10.1016/j.jsbmb.2013.12.010

Matkowski A (2004) In vitro isoflavonoid production in callus from different organs of Pueraria lobata (Wild.) Ohwi. J Plant Physiol 161:343-346. doi:10.1078/0176-1617-01145

Matkowski A (2008) Plant in vitro culture for the production of antioxidants-a review. Biotechnol Adv 26:548-560. doi:10. 1016/j.biotechadv.2008.07.001

Mohamed MF, Read PE, Coyne DP (1992) Dark preconditioning, CPPU and thidiazuron promote shoot organogenesis on seedling node explants of common and faba beans. J Am Soc Hortic Sci 117:668-672

Mourouti N, Panagiotakos DB (2013) Soy food consumption and breast cancer. Maturitas 76:118-122. doi:10.1016/j.maturitas. 2013.07.006
Ozeki Y (1996) Regulation of anthocyanin synthesis in carrot suspension cultured cells. J Plant Res 109:343-351. doi:10.1007/ BF02344483

Pu L-H, Liu Z-H, Wei Z-Y, Wang J-F, Dong Q-Q, Fan Y-L (2013) Callus culture of embryos in Sophora japonica and analysis on isoflavones content. Chin Tradit Herb Drugs 44:1984-1989. doi: $10.7501 /$ j.issn.0253-2670.2013.14.022

Shinde AN, Malpathak N, Fulzele DP (2010) Determination of isoflavone content and antioxidant activity in Psoralea corylifolia L. callus cultures. Food Chem 118:128-132. doi: 10.1016/j. foodchem.2009.04.093

Smetanska I (2008) Production of secondary metabolites using plant cell cultures. Adv Biochem Eng Biotechnol 111:187-228. doi:10.1007/10_2008_103

Stalman M, Koskamp A-M, Luderer R, Vernooy JHJ, Wind JC, Wullems GJ, Croes AF (2003) Regulation of antraquinone biosynthesis in cell cultures of Morinda citrifolia. J Plant Physiol 160:607-614. doi:10.1078/0176-1617-00773

Swedlund B, Locy RD (1993) Sorbitol as the primary carbon source for the growth of embryogenic callus of maize. Plant Physiol 103:1339-1346. doi:10.1104/pp.103.4.1339

Szopa A, Ekiert H (2014) Production of biologically active phenolic acids in Aronia melanocarpa (Michx.) Elliott in vitro cultures cultivated on different variants of the Murashige and Skoog medium. Plant Growth Regul 72:51-58. doi:10.1007/s10725013-9835-2

Szopa A, Ekiert H, Muszyńska B (2013) Accumulation of hydroxybenzoic acids and other biologically active phenolic acids in shoot and callus cultures of Aronia melanocarpa (Michx.) Elliott (black chokeberry). Plant Cell Tissue Organ Cult 113:323-329. doi:10.1007/s11240-012-0272-0

Tůmová L, Tůma J (2011) The effect of UV light on isoflavonoid production in Genista tinctoria culture in vitro. Acta Physiol Plant 33:635-640. doi:10.1007/s11738-010-0566-y

Tůmová L, Šárková T, Dušek J (2007) Genista tinctoria in vitro. Ceska Slov Farm 56:21-26

Udomsuk L, Jarukamjorn K, Tanaka H, Putalun W (2009) Production of isoflavonoids in callus cultures of Pueraria candollei var. mirifica. Z Naturforsch 64c:239-243

Udomsuk L, Juengwattanatrakul T, Jarukamjorn K, Putalun W (2012) Increased miroestrol, deoxymiroestrol and isoflavonoid accumulation in callus and cell suspension cultures of Pueraria candollei var. mirifica. Acta Physiol Plant 34:1093-1100. doi:10.1007/s11738-011-0906-6

Vaishnav K, Goyal S, Ramawat KG (2006) Isoflavonoids production in callus culture of Pueraria tuberosa, the Indian kudzu. Indian J Exp Biol 44:1012-1017

van Die MD, Bone KM, Williams SG, Pirotta MV (2014) Soy and soy isoflavones in prostate cancer: a systematic review and metaanalysis of randomised controlled trials. BJU Int. doi:10.1111/ bju. 123435

Zhao L, Mao Z, Brinton RD (2009) A select combination of clinically relevant phytoestrogens enhances estrogen receptor $\beta$-binding selectivity and neuroprotective activities in vitro and in vivo. Neuroendocrinology 150:770-783. doi:10.1210/en.2008-0715 